\title{
QTL analysis of measures of mouse home-cage activity using B6/MSM consomic strains
}

\author{
Akinori Nishi · Ayako Ishii · Aki Takahashi • \\ Toshihiko Shiroishi · Tsuyoshi Koide
}

Received: 27 July 2010/ Accepted: 13 September 2010/Published online: 1 October 2010

(C) The Author(s) 2010. This article is published with open access at Springerlink.com

\begin{abstract}
The activity of mice in their home cage is influenced greatly by the cycle of light and dark. In addition, home-cage activity shows remarkable time-dependent changes that result in a prominent temporal pattern. The wild-derived mouse strain MSM/Ms (MSM) exhibits higher total activity in the home cage than does C57BL/6 (B6), a commonly used laboratory strain. In addition, there is a clear strain difference in the temporal pattern of home-cage activity. This study aimed to clarify the genetic basis of strain differences in the temporal pattern of home-cage activity between MSM and B6. Through the comparison of temporal patterns of home-cage activity between B6 and MSM, the pattern can be classified into five temporal components: (1) resting phase, (2) anticipation phase, (3) 1st phase, (4) 2nd phase, and (5) 3rd phase. To identify quantitative trait loci (QTLs) involved in these temporal components, we used consomic strains established from crosses between B6 and MSM. Five consomic strains, for
\end{abstract}

Electronic supplementary material The online version of this article (doi:10.1007/s00335-010-9289-z) contains supplementary material, which is available to authorized users.

A. Nishi · A. Ishii · A. Takahashi $\cdot$ T. Koide $(\bowtie)$

Mouse Genomics Resource Laboratory, National Institute

of Genetics, 1111 Yata, Mishima, Shizuoka 411-0801, Japan

e-mail: tkoide@lab.nig.ac.jp

T. Shiroishi

Mammalian Genetics Laboratory, National Institute of Genetics,

1111 Yata, Mishima, Shizuoka 411-0801, Japan

A. Nishi - A. Ishii · A. Takahashi - T. Shiroishi - T. Koide Department of Genetics, SOKENDAI, Hayama, Kanagawa 240-0193, Japan
Chrs 2T (telomere), 3, 4, 13, and 14, showed significantly higher total activity than B6. In contrast, the consomic strains of Chrs 6C (centromere), 7T, 9, 11, and 15 were less active than B6. This indicates that multigenic factors regulate the total activity. Further analysis showed an impact of QTLs on the temporal components of home-cage activity. The present data showed that each temporal component was regulated by different combinations of multigenic factors, with some overlap. These temporal component-related QTLs are important to understand fully the genetic mechanisms that underlie home-cage activity.

\section{Introduction}

There are two principal ways of measuring general or spontaneous activity in laboratory rodents: the use of running wheels (Koteja et al. 1999; Pittendrigh and Daan 1974) or, as in the present experiment, measurement of activity in the home cage (Kas et al. 2009; Koide et al. 2000). A number of studies have found that spontaneous activity in the laboratory mouse is a quantitative trait that is influenced by multiple genetic factors (Kas et al. 2009; Koyner et al. 2000; Turri et al. 2001). Several experiments conducted in our laboratory on crosses between wildderived strains yielded results that were consistent with this finding. A cross between two wild-derived strains, KJR (characterized by high home-cage activity) and BLG2 (characterized by low home-cage activity), identified two loci on Chr 3 and Chr 17 that are related to the difference in home-cage activity between KJR and BLG2 (Furuse et al. 2002a). Another quantitative trait locus (QTL) analysis of home-cage activity, which used an F2 cross between the strains KJR and B6, identified two linked QTLs on Chr 2 
and one locus on Chr 10 (Umemori et al. 2009). However, home-cage activity varies with the time of day, and the genetic basis of the diversity in the temporal pattern of home-cage activity remains to be clarified.

Consomic strains, which are also known as chromosome substitution strains, have been used as a powerful tool for the systematic genetic analysis of complex traits (Nadeau et al. 2000; Petryshen et al. 2005; Singer et al. 2004, 2005). Recently, a new set of consomic strains, denoted B6$\mathrm{ChrN}^{\mathrm{MSM}}$, in which each chromosome of B6 was replaced by the corresponding chromosome from the strain MSM, has been established (Takada et al. 2008). The strain MSM is derived from wild Japanese mice of the subspecies Mus musculus molossinus and has a high rate of nucleotide polymorphism relative to $\mathrm{B} 6$, which is derived mostly from M. m. domesticus (Abe et al. 2004; Koide et al. 2000; Moriwaki et al. 2009; Sakai et al. 2005). The frequency of polymorphism between these two strains on the basis of single-nucleotide substitutions is 0.0096 (Abe et al. 2004), which is seven times higher than the overall rate of polymorphism between strains 129 and B6 (Wade et al. 2002). Recently, systematic QTL mapping was conducted for a series of behavioral tests related to emotionality and social behavior and for the incidence of hydrocephalus. Multiple loci were mapped for these phenotypes on many chromosomes in the genome (Takahashi et al. 2008a, b, 2010). By taking advantage of using a panel of B6-ChrN ${ }^{\mathrm{MSM}}$ consomic strains, we attempted to conduct systematic genetic mapping of loci related to spontaneous home-cage activity.

\section{Materials and methods}

\section{Animals}

Mice of strains C57BL/6JJcl (B6), MSM/Ms (MSM), and consomic strains derived from B6 and MSM were used for this study. B6 mice were purchased from CLEA Japan, Inc. (Tokyo, Japan) and bred at the animal facility of the National Institute of Genetics (NIG), Mishima, Japan. MSM was established as an inbred strain from Japanese wild mice and maintained at the NIG (Moriwaki et al. 2009). The establishment of the B6-ChrN ${ }^{\mathrm{MSM}}$ consomic panel has been described in detail (Takada et al. 2008). All the consomic strains have the same genetic background as B6, except for one pair of chromosomes that were replaced with the corresponding chromosomes from MSM. It proved difficult to substitute the entire chromosome for Chrs 2, 6, 7 , and 12. Therefore, for each of these chromosomes, two subconsomic strains that carried the telomeric (T) and centromeric (C) portions, respectively, and that together covered the whole chromosome were established. The consomic strains for Chrs 5, 7C, 10, and X could not be characterized in this study because of some difficulties with breeding and analyses. Each consomic strain was described as B6-ChrN ${ }^{\mathrm{MSM}}$, in which $\mathrm{N}$ was the number of the chromosome transferred from the MSM strain.

All the animals were housed with their same-sex littermates in a plastic cage (measuring $19.5 \times 29.5 \times 15 \mathrm{~cm}$ ) which contained wood chips for bedding material and was maintained with a constant temperature of $23 \pm 2^{\circ} \mathrm{C}$ and a 12-h light/dark cycle with lights on at 08:00. Food and water were available ad libitum. The home-cage activity of all mice was tested at 9-12 weeks of age. The mice were maintained according to NIG guidelines, and all procedures were carried out with the approval of our institutional animal care and use committee.

\section{Behavioral testing}

Before the test, mice were kept individually for more than 1 day in their home cage to habituate them to isolation. The home-cage test constituted the recording of individual spontaneous home-cage activity from 08:00 on the day after transfer for 3 days. To evaluate the spontaneous activity of each mouse in their home cage, an infrared sensor (ACTIVITY SENSOR, Ohara Co. Ltd., Tokyo, Japan) was used. This sensor was located above the lid (made from stainless-steel wire) of each cage. The sensor records the motion of the mouse inside the home cage as counts by the sensor. Using this apparatus, many kinds of activity, such as horizontal locomotion, climbing the cage lid, hanging on the lid, and jumping, could be detected efficiently. However, grooming or foraging behavior that occurred in one place was detected less efficiently because we chose the lower-detection-sensitivity mode for this sensor. The test apparatus used can record activity in the home cage for 24 mice simultaneously. In each test session we analyzed up to 24 mice from several different strains at once. Mice of control strain B6 were also separated into several groups and recorded with mice of other strains. The recordings of home-cage activity were carried out by two different investigators using the same protocol. The activity of each 1-min bin was measured by accumulated counts if the animal was active in any area of the cage. The activity counts for $72 \mathrm{~h}$ were summed and the scores of total activity corresponded to the average counts for a 1-day period over 3 days. Total activity was divided into two components: active time and average activity (Umemori et al. 2009). Active time was calculated as the total number of minutes in which the mouse accumulated more than one count within a 1-min interval. Hence, the active time estimated the approximate duration of movement. Average activity, which was an index of "intensity," was calculated 
using the formula: average activity $=$ total activity $\div$ active time. The average activity reflected the average amount of locomotion over $1 \mathrm{~min}$ of active time.

For the analyses of temporal patterns, average activity counts for each 1-h bin over the 3 days were calculated and used as scores for each hour.

\section{Statistical analysis}

Statistical analyses of the behavioral test data were performed using the Statview software package (SAS Institute Inc, Cary, NC, USA).

\section{Comparison of parental strains}

Two-way analysis of variance (ANOVA) between-groups (strain $\times$ sex) was performed to compare the total activity, active time, and average activity of B6 and MSM. In the case of significant $F$ values, Tukey-Kramer's $t$ test was conducted. To analyze the temporal pattern of activity, a three-way ANOVA was used (strain $\times \operatorname{sex} \times$ repeated measures over time).

\section{Comparison of consomic strains}

One-way between-groups ANOVA (strain) was performed to compare the total activity, active time, and average activity of the B6 and consomic strains. In the case of significant $F$ values, a $t$ test with Bonferroni correction $[\alpha=0.05 / 19$ (number of consomic strains) $]$ was conducted to compare B6 with each consomic strain. For comparison, $Z$ scores were also estimated using Eq. 3 from Belknap (2003), which is a standard method used to examine the effect of consomic strains (Supplementary Table 1). In this article we used results obtained with the Bonferroni correction as the results were more conservative than were those from Belknap's method. To compare hour-by-hour activity, repeated two-way ANOVA (strain $\times$ time) was conducted. Each 24-h session was divided into five temporal components: (1) resting phase (10:00-17:59); (2) phase of anticipation of the dark cycle: anticipation phase (18:0019:59); (3) burst of activity at the beginning of the dark cycle: 1st phase (20:00-0:59); (4) MSM-specific activity peak: 2nd phase (01:00-05:59); and (5) activity peak at the end of the dark cycle: 3 rd phase (06:00-09:59). The average activity counts for each time period over 3 days were
(A)

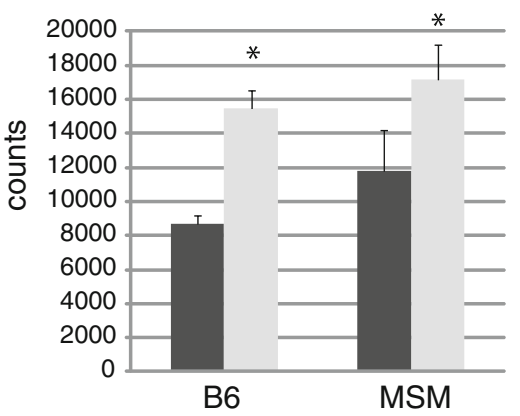

(B)

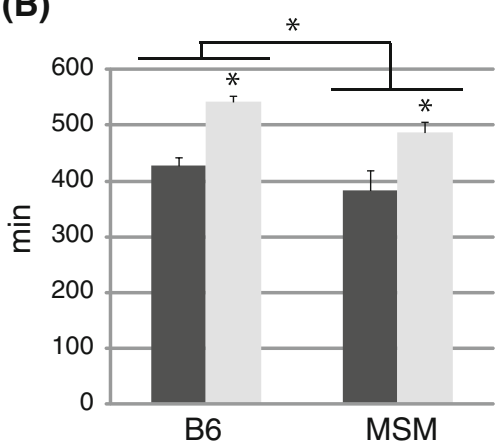

(C)

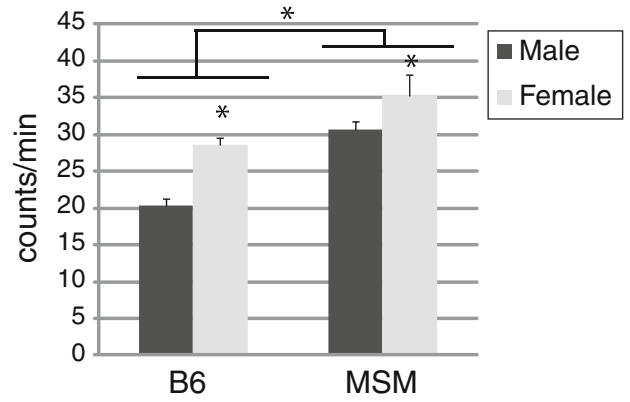

(D)

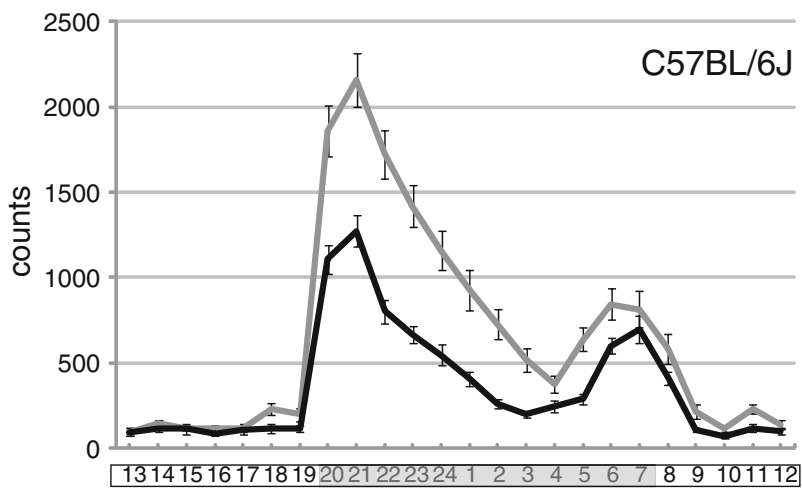

Fig. 1 Comparison of home-cage activity between B6 and MSM mice. a Total activity. b Active time. c Average activity. Error bars indicate SEM in (a), (b), and (c). Asterisks indicate significant

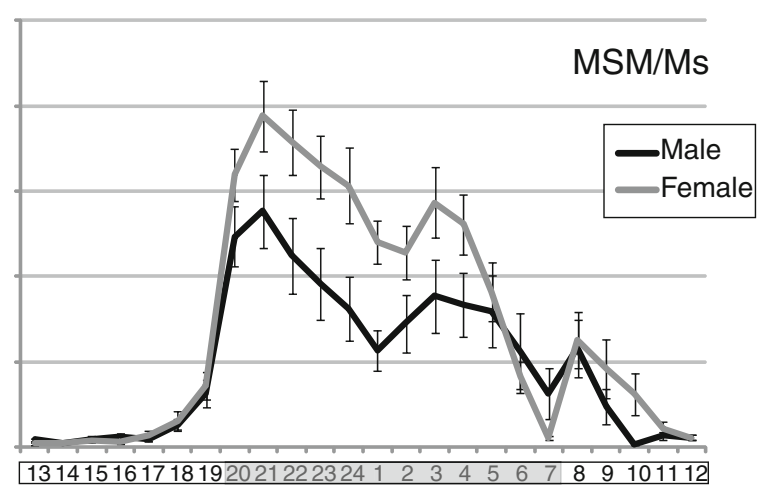

difference $(P<0.05)$. Asterisks on the female bars indicate significant difference between sexes. d Temporal pattern of home-cage activity 
calculated and presented as scores for each temporal component. One-way between-groups ANOVA was performed to compare the activity in each temporal component. A $t$ test with Bonferroni correction $[\alpha=0.05 / 19$ (number of consomic strains)] was conducted as a post-hoc test to compare B6 with each consomic strain. In addition, $Z$ scores were estimated using Belknap's method (Supplementary Table 2).

\section{Results}

Comparison of parental strains

The home-cage activity of B6 and MSM was examined in both males and females (Fig. 1). Two-way ANOVA showed a significant effect of strain with respect to active time $[F(1,90)=6.750, P=0.011]$ and average activity $[F(1,90)=9.7338, P=0.002]$, and a trend with respect to total activity $[F(1,90)=3.145, P=0.080]$. No strain $\times$ sex interaction was detected for any of the three indices. Post-hoc analyses demonstrated that B6 showed a longer active time than MSM (Fig. 1b), whereas MSM had higher total activity (Fig. 1a) and average activity than B6 (Fig. 1c). The temporal pattern of home-cage activity (hour-by-hour) of B6 and MSM is indicated in Fig. 1d. Interestingly, males and females within the same strain showed consistent temporal patterns of home-cage activity: B6 had two peaks of activity during the dark period, whereas MSM was characterized by three peaks of activity and also an anticipatory increase in activity right before the beginning of the dark period. Three-way ANOVA showed that all the interactions were significant (strain $\times$ time $[F(23,2070)=15.738, P<0.001]$; sex $\times$ time $[F(23,2070)=$ 13.791, $P<0.001]$; strain $\times$ sex $\times$ time $[F(23,2070)=1.772$, $P=0.018])$. Significance was detected in the three-way interactions as a result of the higher activity of the females during the dark period. By inspection, males and females of the same strain had a similar temporal pattern (peak structure) but a different basal level of activity (Fig. 1d).

Chromosomal mapping using consomic strains

To identify the chromosomes that affected home-cage activity, we used males of 19 consomic strains established from B6 and MSM (Table 1). One-way ANOVA showed the significant main effect of strain in the total activity $[F(19$, $335)=13.632, P<0.001]$, the active time $[F(19,335)=$ $16.657, P<0.001]$, and the average activity $[F(19,335)=$ $10.371, P<0.001]$. We found exactly the same pattern of strain differences for total activity and average activity
Table 1 Strains and number of animals used in this study

\begin{tabular}{|c|c|c|c|}
\hline Strains & & Male & Female \\
\hline \multicolumn{4}{|l|}{ Parental strains } \\
\hline B6 & & 34 & 27 \\
\hline MSM & & 16 & 17 \\
\hline Consomic name & Chr & & \\
\hline B6-Chr1 ${ }^{\mathrm{MSM}}$ & 1 & 17 & \\
\hline B6-Chr2C & $2 \mathrm{C}$ & 15 & \\
\hline B6-Chr2T ${ }^{\mathrm{MSM}}$ & $2 \mathrm{~T}$ & 17 & \\
\hline B6-Chr3 ${ }^{\mathrm{MSM}}$ & 3 & 21 & \\
\hline B6-Chr4 ${ }^{\mathrm{MSM}}$ & 4 & 15 & \\
\hline B6-Chr6C ${ }^{\mathrm{MSM}}$ & $6 \mathrm{C}$ & 18 & \\
\hline B6-Chr7T ${ }^{\mathrm{MSM}}$ & $7 \mathrm{~T}$ & 19 & \\
\hline B6-Chr8 ${ }^{\mathrm{MSM}}$ & 8 & 16 & \\
\hline B6-Chr9 $9^{\mathrm{MSM}}$ & 9 & 18 & \\
\hline B6-Chr11 MSM & 11 & 15 & \\
\hline B6-Chr12C ${ }^{\mathrm{MSM}}$ & $12 \mathrm{C}$ & 17 & \\
\hline B6-Chr12T ${ }^{\mathrm{MSM}}$ & $12 \mathrm{~T}$ & 16 & \\
\hline B6-Chr13A ${ }^{\text {MSM }}$ & 13 & 16 & \\
\hline B6-Chr14 ${ }^{\mathrm{MSM}}$ & 14 & 16 & \\
\hline B6-Chr15 ${ }^{\mathrm{MSM}}$ & 15 & 20 & \\
\hline B6-Chr16 ${ }^{\mathrm{MSM}}$ & 16 & 15 & \\
\hline B6-Chr17 ${ }^{\mathrm{MSM}}$ & 17 & 15 & \\
\hline B6-Chr19 ${ }^{\text {MSM }}$ & 19 & 16 & \\
\hline B6-ChrY ${ }^{\mathrm{MSM}}$ & $\mathrm{Y}$ & 19 & \\
\hline
\end{tabular}

$(r=0.964)$ (data not shown), and thus we excluded the average activity from further analysis.

\section{Total activity}

Consomic strains that contained MSM-derived Chrs 2T, 3, 4, 13, and 14 showed increased total activity compared with B6 (Fig. 2a). Reduced activity in comparison with B6 was observed in the consomic strains of Chrs 6C, 7T, 9, 11, and 15.

\section{Active time}

Increased active time compared with that of B6 was observed in the consomic strains that contained MSMderived Chrs 3, 4, 12T, and 13 (Fig. 2b). Three consomic strains, those of Chrs 9, 11, and 15, showed decreased active time compared with that of B6.

\section{Temporal pattern}

Figure $3 \mathrm{a}$ and $\mathrm{b}$ shows the temporal pattern of home-cage activity in males of B6, MSM, and each consomic strain. Comparison of B6 and MSM (Fig. 3a, top left) showed 
Fig. 2 Results of systematic analysis using consomic strains. All data were obtained from male mice. Horizontal dashed line indicates the level of B6. Asterisks indicate significant increase or reduction of the scores in comparison with B6 $(P$ value with Bonferroni correction: $P<0.05 /$ $19=0.00263)$. Means, SEM, and $P$ values are indicated in Supplementary Table 1. a Results of comparison of total activity. b Results of comparison of active time

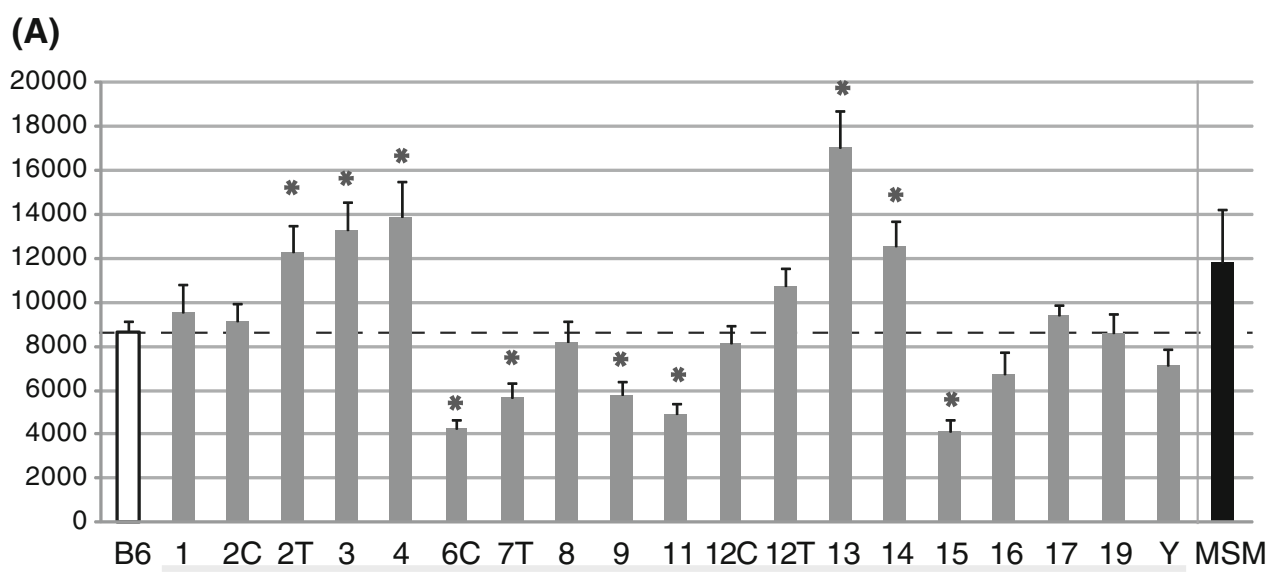

(B)

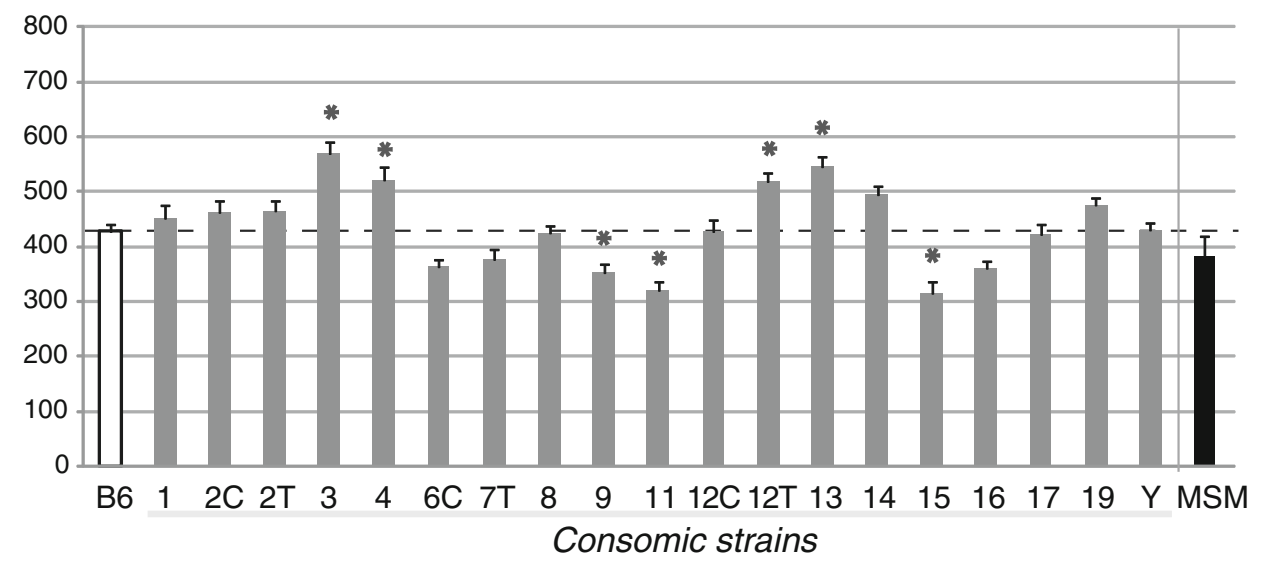

Table 2 Chromosomal mapping of loci related to temporal components of home-cage activity

\begin{tabular}{|ll|l|l|l|l|l|l|l|l|l|l|l|l|l|l|l|l|l|l|l||}
\hline Phase Time & 1 & $2 \mathrm{C}$ & $2 \mathrm{~T}$ & 3 & 4 & $6 \mathrm{C}$ & $7 \mathrm{~T}$ & 8 & 9 & 11 & $12 \mathrm{C}$ & $12 \mathrm{~T}$ & 13 & 14 & 15 & 16 & 17 & 19 & $\mathrm{Y}$ & $\mathrm{MSM}$ \\
\hline Anticipation (18-19) & & & & & & & & & & & & & & & & & & & & \\
\hline 1st phase (20-24) & & & & & & & & & & & & & & & & & & & & \\
\hline 2nd phase (1-5) & & & & & & & & & & & & & & & & & & & & \\
\hline 3rd phase (6-9) & & & & & & & & & & & & & & & & & & & & \\
\hline Resting phase (10-17) & & & & & & & & & & & & & & & & & & & & \\
\hline
\end{tabular}

Filled cells are strains that showed significantly higher (gray) or lower (black) values than the parental B6 strain in one component Significant $P$ value with Bonferroni correction: $P<0.05 / 19=0.00263$. Means, SEM, and $P$ values are indicated in Supplementary Table 2

prominent differences in peak structures between these parental strains. MSM mice showed lower activity than B6 during most of the light period (resting), whereas they started to increase their activity 1 hour before the beginning of the dark period (anticipation). Both B6 and MSM showed the highest peak of activity during the first $2 \mathrm{~h}$ of the dark period (1st peak), and their level of activity then decreased gradually. MSM mice had a second increase (2nd peak) of activity in the middle of the dark period, whereas B6 mice were inactive during this period. At the end of the dark period or beginning of the light period, both
B6 and MSM mice showed an increase in activity (3rd peak). Therefore, we decided to define five temporal components (the resting phase, anticipation phase, 1st phase, 2nd phase, and 3rd phase) and to compare strain B6 and the consomic strains with respect to these components (Table 2). One-way ANOVA showed a significant main effect of strain for all temporal components $[F(19$, $335) \geq 7.030, P<0.001]$. The consomic strains of Chrs 1 , 3,13 , and 14 showed an increase of activity during the anticipation phase, but B6-Chr6C ${ }^{\mathrm{MSM}}$ showed a significant decrease. Although the 1st phase did not differ between the 
parental strains B6 and MSM, the consomic strains of Chrs 2T, 4, and 13 showed an increase and those of Chrs 6C, 7T, 9,11 , and 15 showed a reduction in activity. In the 2 nd phase, Chrs 3, 4, 12T, 13, and 14 showed an increase but Chrs 6C, 7T, 9, 11, and 15 showed a decrease. Although the 3rd phase was not significantly different between the parental strains, the consomic strains of Chrs 6C, 7T, 8, 11, 15 , and $\mathrm{Y}$ showed a significant reduction. During the resting period, Chrs $6 \mathrm{C}, 11$, and 15 showed significantly lower activity than the parental MSM strain, but Chrs 1 and 13 showed higher activity. Therefore, the results showed that each temporal component was affected by different QTLs.

\section{Discussion}

Previous studies have shown that MSM and B6 are characterized by marked differences in several behavioral traits (Furuse et al. 2002b; Koide et al. 2000; Takahashi et al. 2006). In this study we found that MSM mice showed less active time but higher average activity in the home cage than B6 mice. We also found a lower level of activity in males than in females for both B6 and MSM mice, which agreed with the results of a previous report that used B6 mice (Kas et al. 2009). Although there was a clear sex difference in total activity, the temporal patterns of homecage activity were very similar in males and females for both B6 and MSM mice. This result showed that homecage activity was higher in females than in males but the genetic mechanism of temporal regulation of activity did not differ between the two sexes. Therefore, we decided to focus only on males in the further genetic analysis of home-cage activity.

The temporal pattern of home-cage activity clearly differed between B6 and MSM mice. The differences in this pattern led to the classification of the temporal pattern into five components: resting phase, anticipation phase, 1st phase, 2nd phase, and 3rd phase. This classification allowed us to identify three additional chromosomes, 1, 8, and $\mathrm{Y}$, that were overlooked when we used only parameters such as total activity or active time. By adopting the five components, QTLs related to strain differences in homecage activity could be detected with greater sensitivity.

An anticipatory increase in activity has been reported also in other laboratory inbred strains of mice (Kopp 2001). For example, $\mathrm{C} 3 \mathrm{H} / \mathrm{HeJ}$ and $\mathrm{CBA} / \mathrm{J}$ mice display an increase in activity a few hours before the onset of darkness, whereas the activity of B6 and BALB mice does not change until the dark cycle has started. Interestingly, the latter strains show rapid adaptation to phase shifts of the light-dark cycle, and they also show a deficiency in
Fig. 3 Comparison of temporal pattern of home-cage activity between B6 and MSM or consomic strains. a, b All data were obtained from male mice. Dark and light phases are shown under each graph. Each temporal phase is marked by monotone background colors to distinguish from other phases. $R$ resting phase, $A$ anticipation phase, 1st 1st phase, 2nd 2nd phase, 3rd 3rd phase

the synthesis of melatonin (Vivien-Roels et al 1998). Therefore, this anticipatory change in activity might reflect circadian rhythmicity; however, this needs to be examined further. In this study we identified genetic loci involved in this anticipatory increase of activity on Chrs 1, 3, 13, and 14. We dissected the nocturnal activity into three components. B6 mice showed two peaks of activity, at the beginning and the end of the dark period, which was consistent with a previous report (Mayeda and Hofstetter 1999). In contrast, the pattern of peaks of activity for MSM mice was different; they showed an increase in activity during the middle of the dark period (02:00-05:00), in addition to the first and last peaks that were characteristic of B6. Use of the consomic strains showed that these peaks were influenced by different genetic components. It is essential to consider the effects specific to different temporal components in the analysis of the genetic basis of the regulation of home-cage activity.

The results of the present study showed that 14 of 19 strains demonstrated a difference in one or more temporal components of home-cage activity. Turri et al. (2001) conducted QTL mapping of home-cage activity by measuring activity during the first $2 \mathrm{~h}$ of the dark period and found six chromosomes $(4,8,11,14,18$, and $\mathrm{X})$ that had an effect on the level of activity. The measure of activity that they used was similar to the 1 st phase component (1st $4 \mathrm{~h}$ of the dark period) in our current study. The results showed that QTLs on two chromosomes, 4 and 11, overlapped between the two studies, but QTLs on eight chromosomes, 2T, 6C, 7T, 8, 9, 13, 14, and 15, did not overlap (we did not study chromosomes 18 and $\mathrm{X}$ in the current study). In previous QTL studies using another wild-derived strain, KJR, we identified four chromosomes (2, 3, 10, and 17) that were related to home-cage activity (Furuse et al. 2002a; Umemori et al. 2009). These studies indicate that home-cage activity is influenced by multigenic factors and is regulated differently, with some overlap, in different crosses.

In order to analyze the QTLs in detail, we are currently establishing subconsomic strains for some of the chromosomes. Through the study of subconsomic strains we will be able to obtain more precise mapping information on the genes related to different levels of home-cage activity. By studying the smaller regions available in these recombinant strains, it might be possible to conduct molecular studies of behavioral traits in the near future. 
(A)
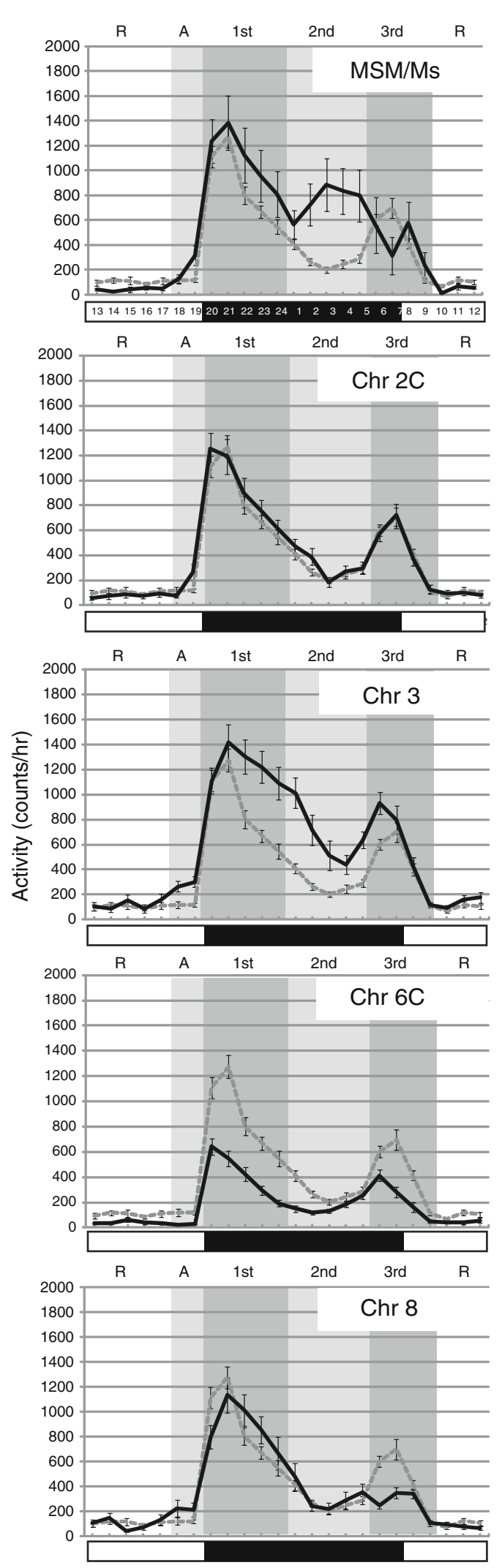

..- C57BL/6J

- Consomic strain
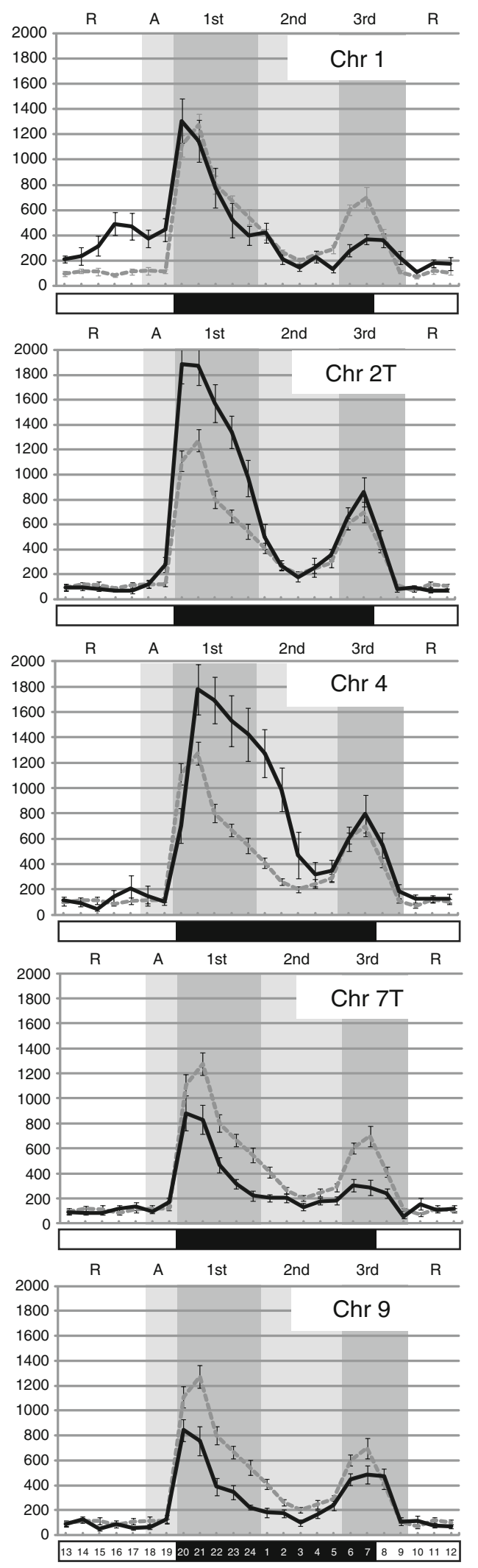
(B)
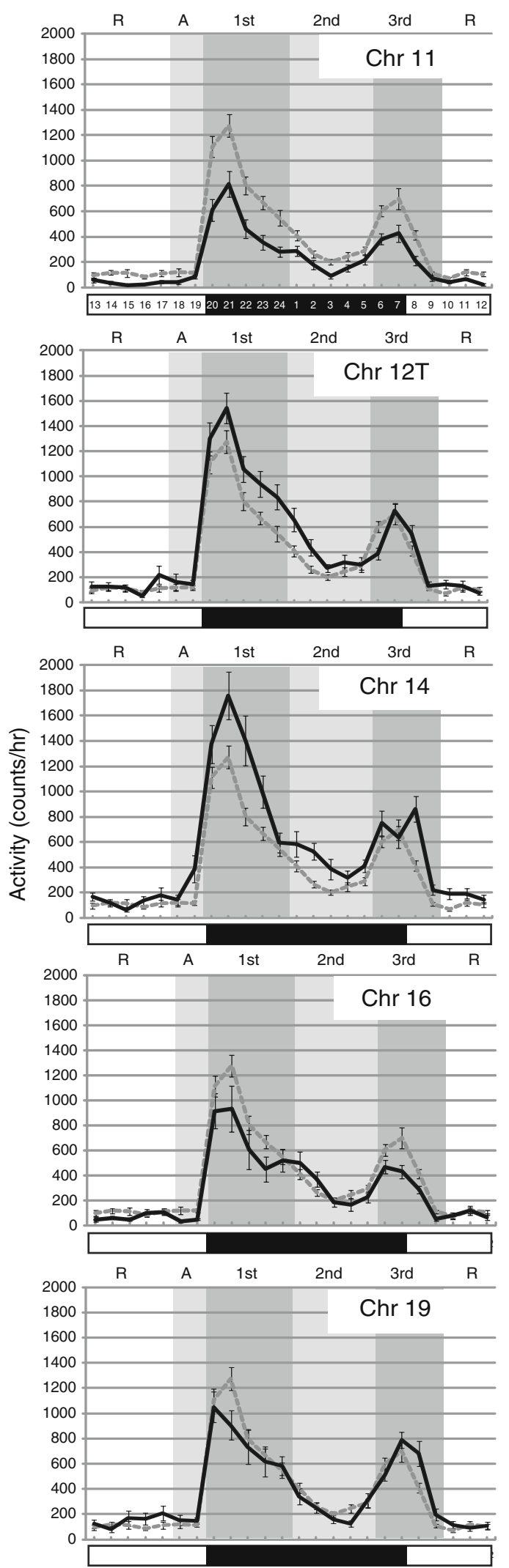

-.. C57BL/6J

- Consomic strain
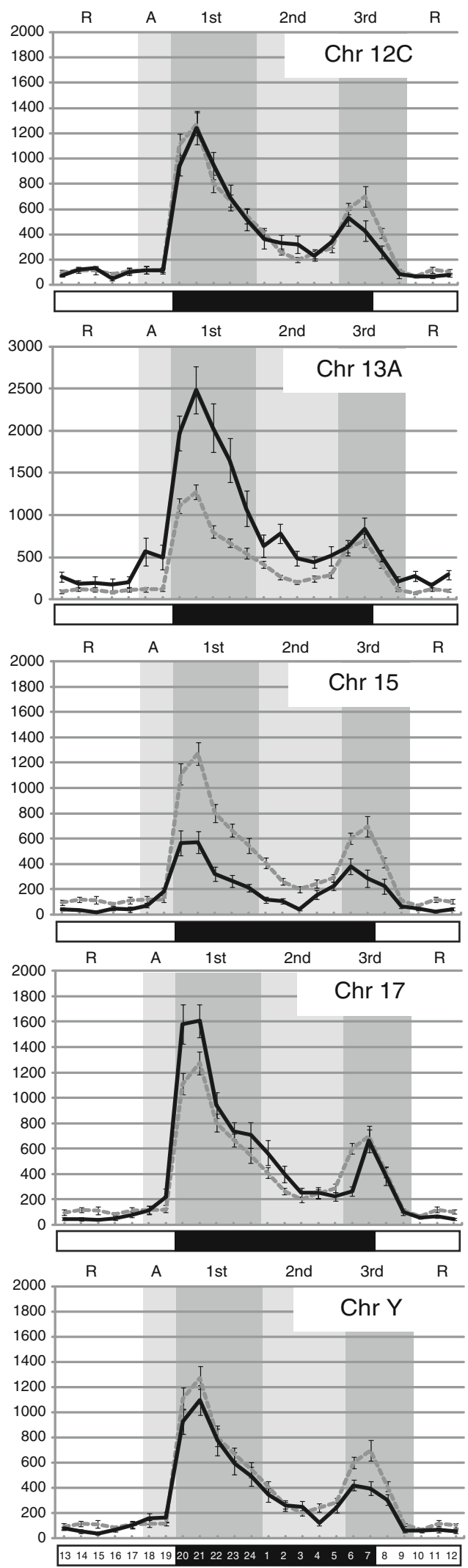

Fig. 3 continued 
Acknowledgments The authors thank K. Moriwaki for his great contribution in establishing the MSM strain and D. A. Blizard for critical comments on the manuscript. This work was supported by KAKENHI (Grant-in-Aid for Scientific Research) from the Ministry of Education, Culture, Sports, Science and Technology of Japan, the Japan Society for the Promotion of Science (JSPS), and the Research Organization of Information and Systems, Transdisciplinary Research Integration Center.

Open Access This article is distributed under the terms of the Creative Commons Attribution Noncommercial License which permits any noncommercial use, distribution, and reproduction in any medium, provided the original author(s) and source are credited.

\section{References}

Abe K, Noguchi H, Tagawa K, Yuzuriha M, Toyoda A et al (2004) Contribution of Asian mouse subspecies Mus musculus molossinus to genomic constitution of strain C57BL/6J, as defined by BAC-end sequence-SNP analysis. Genome Res 14:2439-2447

Belknap JK (2003) Chromosome substitution strains: some quantitative considerations for genome scans and fine mapping. Mamm Genome 14:723-732

Furuse T, Takano-Shimizu T, Moriwaki K, Shiroishi T, Koide T (2002a) QTL analyses of spontaneous activity by using mouse strains from Mishima battery. Mamm Genome 13:411-415

Furuse T, Blizard DA, Moriwaki K, Miura Y, Yagasaki K et al (2002b) Genetic diversity underlying capsaicin intake in the Mishima battery of mouse strains. Brain Res Bull 57:49-55

Kas MJ, de Mooij-van Malsen JG, de Krom M, van Gassen KL, van Lith HA et al (2009) High-resolution genetic mapping of mammalian motor activity levels in mice. Genes Brain Behav 8: $13-22$

Koide T, Moriwaki K, Ikeda K, Niki H, Shiroishi T (2000) Multiphenotype behavioral characterization of inbred strains derived from wild stocks of Mus musculus. Mamm Genome 11:664-670

Kopp C (2001) Locomotor activity rhythm in inbred strains of mice: implications for behavioural studies. Behav Brain Res 125:93-96

Koteja P, Garland T Jr, Sax JK, Swallow JG, Carter PA (1999) Behaviour of house mice artificially selected for high levels of voluntary wheel running. Anim Behav 58:1307-1318

Koyner J, Demarest K, McCaughran J Jr, Cipp L, Hitzemann R (2000) Identification and time dependence of quantitative trait loci for basal locomotor activity in the BXD recombinant inbred series and a B6D2 F2 intercross. Behav Genet 30:159-170

Mayeda AR, Hofstetter JR (1999) A QTL for the genetic variance in free-running period and level of locomotor activity between inbred strains of mice. Behav Genet 29:171-176
Moriwaki K, Miyashita N, Mita A, Gotoh H, Tsuchiya K et al (2009) Unique inbred strain MSM/Ms established from the Japanese wild mouse. Exp Anim 58:123-134

Nadeau JH, Singer JB, Matin A, Lander ES (2000) Analysing complex genetic traits with chromosome substitution strains. Nat Genet 24:221-225

Petryshen TL, Kirby A, Hammer RP Jr, Purcell S, O'Leary SB et al (2005) Two quantitative trait loci for prepulse inhibition of startle identified on mouse chromosome 16 using chromosome substitution strains. Genetics 171:1895-1904

Pittendrigh CS, Daan S (1974) Circadian oscillations in rodents: a systematic increase of their frequency with age. Science 186: $548-550$

Sakai T, Kikkawa Y, Miura I, Inoue T, Moriwaki K et al (2005) Origins of mouse inbred strains deduced from whole-genome scanning by polymorphic microsatellite loci. Mamm Genome 16:11-19

Singer JB, Hill AE, Burrage LC, Olszens KR, Song J et al (2004) Genetic dissection of complex traits with chromosome substitution strains of mice. Science 304:445-448

Singer JB, Hill AE, Nadeau JH, Lander ES (2005) Mapping quantitative trait loci for anxiety in chromosome substitution strains of mice. Genetics 169:855-862

Takada T, Mita A, Maeno A, Shitara H, Kikkawa Y et al (2008) Mouse inter-subspecific consomic strains for genetic dissection of quantitative complex traits. Genome Res 18:500-508

Takahashi A, Kato K, Makino J, Shiroishi T, Koide T (2006) Multivariate analysis of temporal descriptions of open-field behavior in wild-derived mouse strains. Behav Genet 36:763-774

Takahashi A, Nishi A, Ishii A, Shiroishi T, Koide T (2008a) Systematic analysis of emotionality in consomic mouse strains established from C57BL/6J and wild-derived MSM/Ms. Genes Brain Behav 7:849-858

Takahashi A, Shiroishi T, Koide T (2008b) Multigenic factors associated with a hydrocephalus-like phenotype found in intersubspecific consomic mouse strains. Mamm Genome 29:333-338

Takahashi A, Tomihara K, Shiroishi T, Koide T (2010) Genetic mapping of social interaction behavior in B6/MSM consomic mouse strains. Behav Genet 40:366-376

Turri MG, Datta SR, DeFries J, Henderson ND, Flint J (2001) QTL analysis identifies multiple behavioral dimensions in ethological tests of anxiety in laboratory mice. Curr Biol 11:725-734

Umemori J, Nishi A, Lionikas A, Sakaguchi T, Kuriki S et al (2009) QTL analyses of temporal and intensity components of homecage activity in KJR and C57BL/6J strains. BMC Genet 10:40

Vivien-Roels B, Malan A, Rettori MC, Delagrange P, Jeanniot JP et al (1998) Daily variations in pineal melatonin concentrations in inbred and outbred mice. J Biol Rhythms 13:403-409

Wade CM, Kulbokas EJ 3rd, Kirby AW, Zody MC, Mullikin JC et al (2002) The mosaic structure of variation in the laboratory mouse genome. Nature 420:574-578 